Service social

\title{
La recherche-action à caractère militant : le cas du GRAP
}

\section{Denis Fortin}

Volume 34, numéro 2-3, 1985

L’organisation communautaire

URI : https://id.erudit.org/iderudit/706272ar

DOI : https://doi.org/10.7202/706272ar

Aller au sommaire du numéro

Éditeur(s)

École de service social de l'Université Laval

ISSN

1708-1734 (numérique)

Découvrir la revue

Citer cet article

Fortin, D. (1985). La recherche-action à caractère militant : le cas du GRAP.

Service social, 34(2-3), 269-293. https://doi.org/10.7202/706272ar d'utilisation que vous pouvez consulter en ligne.

https://apropos.erudit.org/fr/usagers/politique-dutilisation/ 
FORTIN, Denis, professeur à l'École de service social de l'Université Laval.

\section{La recherche-action à caractère militant : le cas du GRAP}

\section{Denis Fortin}

Au terme de presque dix années d'une pratique collective de recherche-action à caractère militant, il fallait bien que l'un des nôtres prenne enfin l'initiative de retracer une partie du chemin parcouru, pour en témoigner et en partager le sens avec d'autres. C'est l'intention du présent texte, dont le cheminement s'effectuera en cinq étapes principales.

Nous évoquerons d'abord certains éléments significatifs de l'histoire du Groupe de recherche en action populaire (GRAP) afin de situer le cadre plus général de cette aventure collective. Cela nous amènera ensuite à examiner la forme et l'organisation du chantier des recherches menées en son sein depuis les années 1975-1976.

Dans un troisième temps, la lunette d'approche sera plus spécifiquement braquée sur la dimension qui nous a le plus directement impliqué, soit le secteur de recherche portant sur la protection des consommateurs. À cette occasion, nous scruterons de manière plus attentive la question du rapport dialectique d'échange entre la recherche et l'action, dont la forme en spécifie le caractère militant.

La quatrième partie, plus théorique, nous entraînera dans une discussion portant sur certaines conditions et le sens de la rechercheaction à caractère militant. Nous nous inspirerons à cette fin du vécu de notre pratique au sein du GRAP et d'une typologie de la recherche sociologique élaborée par Colette Humbert (Annexe 3).

Nous conclurons, finalement, sur un espace plus large : la pratique sociologique. Ce sera alors l'occasion pour nous de prendre position sur celle-ci, du moins dans les termes de son ambition de vouloir constituer une contribution significative au développement de la praxis sociale libératrice. 


\section{Le GRAP : une praxis de presque dix années}

Tout a commencé en 1975, à l'initiative d'un collègue professeur en organisation communautaire à l'école de service social de l'Université Laval, Gérald Doré. À l'époque, celui-ci était également étudiant au doctorat en sociologie, et en rédaction de thèse.

Sur son invitation, deux de ses collègues professeurs en organisation communautaire accepteront rapidement de nouer solidarité autour du projet de recherche antérieurement élaboré par celui-ci pour sa thèse doctorale. II s'agit de Vicente De Paula Faleiros, professeur brésilien arrivé au Québec en 1974 comme réfugié politique, exilé du Chili à la suite du coup d'État militaire de 1973, et de l'auteur du présent article.

Pour les trois membres du noyau initial, cette démarche collective de recherche voulait d'abord répondre à un certain nombre d'interrogations fondamentales portant sur les luttes populaires menées dans le champ de la politique sociale, ou encore des conditions de vie, au cours des dernières années.

Au cour de ces interrogations se profilait la question suivante: "Les luttes populaires, qui sont souvent menées avec tant d'acharnement et d'énergies militantes: "quosse ça donne" concrètement ?".

Cette question, après plus de dix années de luttes populaires auxquelles les chercheurs avaient été associés plus ou moins directement, était devenue une préoccupation centrale, tant au niveau des noyaux de militants intellectuels qu'au sein des groupes populaires eux-mêmes.

De nombreux événements, dont entre autres l'infiltration de militants de groupements marxistes-léninistes et la disparition de la Compagnie des jeunes canadiens (C.J.C.), avaient alors contribué à plonger les groupes populaires dans un climat de grand défaitisme, tout en les privant des ressources matérielles et humaines souvent essentielles pour leur survie.

L'heure était alors aux bilans critiques. Il était en effet devenu impérieux, pour la survie des groupes et pour le développement de leurs luttes, de comprendre le pourquoi de tant de désillusions, de tant d'échecs, du moins en apparence, et de cerner les causes d'une démobilisation devenue quasiment endémique.

Cette conjoncture donnera naissance à l'idée des grands sommets populaires, qui seront tenus à Montréal et à Québec dans les années qui suivront. C'est également dans le creuset de cette conjoncture que naîtra le GRAP et que s'élaboreront les plans de ce qui allait devenir, dans les années suivantes, son chantier de recherche. 
Mais l'argent constituant, dans notre cas comme pour les autres, le nerf de la guerre, la question du financement des opérations de la recherche devint dès le départ une préoccupation centrale. Connaissant bien les rigidités des sources traditionnelles de financement en ce qui concerne la recherche de "gauche" ou militante, la traduction conceptuelle du projet initial s'imposa alors comme une nécessité tactique.

Parler de "l'impact des associations volontaires de politique sociale" permit alors de traduire le concept de "luttes populaires menées sur le front des conditions de vie» dans un langage aux allures neutralistes, répondant bien aux exigences pseudo-objectives de l'appareil technocratique et politique chargé de statuer sur notre projet.

Le financement obtenu, un attaché de recherche à temps plein, Marc Roland, se joignait au noyau des trois professeurs-chercheurs, et ce pour la plus grande partie de la durée de la subvention, c'est-à-dire de 1976 à 1981.

Puis, au fil du parcours et pour des périodes plus ou moins longues, un total de cinq étudiantes ou étudiants à la maîtrise à l'École se joindront également à l'équipe de base. Il s'agit de Michèle Arcand, Pierre Fournier, Denis Plamondon, Carole Roberge et Denise Ventelou.

En plus de contribuer à l'œuvre collective, ceux-ci trouveront, au sein du GRAP, l'occasion d'y produire leur thèse ou leur essai. Ils y découvriront également un lieu d'échange parfois très intense, rarement unanime, et l'occasion de vivre certaines solidarités tissées au fil du temps et du travail partagé.

Finalement, l'obtention de deux congés de perfectionnement pour études doctorales en sociologie, l'un par Gérald Doré (de 1976 à 1978) et l'autre par nous-même (de 1977 à 1980), allait fort heureusement ajouter au bassin des ressources du GRAP une force de travail indispensable pour la réalisation du chantier prévu.

\section{L'organisation et la forme du chantier de la recherche}

À l'origine, les trois membres du noyau du GRAP s'étaient entendus sur le sens de leur démarche commune et en avaient précisé les termes de la façon suivante:

"Le projet vise à évaluer l'impact de l'action d'associations volontaires du champ de la politique sociale, dans une perspective de comparaison entre divers secteurs de politique sociale et divers types de pratiques propres à ces associations. 
"Le modèle d'analyse que nous proposons aborde l'action de ces associations comme produit social, sous deux angles complémentaires: celui de la redistribution et celui de la rétroaction sur le phénomène associationniste lui-même.

"Nous entendons ici la redistribution sous un double aspect économique et social.

$[\ldots]$

"La rétroaction sur le phénomène associationniste lui-même nous intéresse à la fois en termes d'autonomie, de liaison (solidarité) et de mobilisation." 1

Par delà la stricte production de connaissances utiles pour les fins de leur enseignement en organisation communautaire, l'intention visée au sein du GRAP consistait à mettre au service des groupes concernés et de d'autres les résultats de leurs travaux. L'objectif ultime était de contribuer à supporter leur réflexion par rapport aux conditions de développement de leur pratique d'organisation et de lutte, et ce, dans le contexte difficile des années 1975-1980 où les choix à faire n'avaient rien d'évident, et où l'inspiration des leçons du passé récent de "nos luttes» pouvait s'avérer fort précieuse.

C'est sur cette base première que le chantier s'élabora et qu'il prit forme lentement au fil des ans. Trois secteurs de la politique sociale avaient été retenus au départ aux fins du projet, ceux-ci étant en correspondance avec les intérêts de recherche et d'implication militante des trois professeurs qui constituaient le noyau initial; ce sont : le LOGEMENT, la SÉCURITÉ DU REVENU et la PROTECTION DES CONSOMMATEURS.

Cette division opérationnelle du chantier et de l'objet de la recherche comportait un triple avantage : répondre aux intérêts et aux affinités de chacun d'entre nous, couvrir un assez large éventail des luttes populaires menées dans le champ de la politique sociale et, finalement, établir la base d'une forme d'organisation du travail qui soit efficace, compte tenu des limites imposées par les conditions matérielles de production et de l'inégal développement de la connaissance dans chacun des secteurs retenus.

Gérald Doré, comme il se devait étant donné ses antécédents de recherche et de militance, prit la responsabilité des opérations du secteur logement. Le collègue Faleiros assuma, quant à lui, le secteur de la sécurité du revenu et nous, celui de la protection des consommateurs, secteur où nous étions déjà étroitement lié sur le plan de notre pratique militante. Nous y reviendrons plus en détail ci-après sous la rubrique suivante. 
C'est donc dans ce cadre général qu'ont été élaborées les différentes productions du GRAP, auxquelles un total de neuf chercheurs, professeurs, attachés de recherche et étudiants, ont été associés à un moment ou l'autre de l'aventure, ainsi que nous l'avons signifié précédemment.

Si nous tentons maintenant de représenter les configurations de l'ensemble du chantier au stade actuel de son développement, après quelque dix années de travail, cela ressemblera au schéma qui suit.

Ce qui a été retenu, pour la construction de cette illustration, c'est l'ensemble des onze cahiers publiés par le GRAP jusqu'à ce jour, et dont nous retrouvons la liste à l'annexe 1 du présent texte.

Il faut savoir, à cet égard, que ces cahiers de vulgarisation, qui comptent en moyenne quelque trente pages chacun, ne constituent pas la production totale réalisée au sein du GRAP au cours de toutes ces années. En effet, à la base de chacun d'eux il y a une recherche élaborée, ou bien sous la forme d'une thèse de doctorat, ou bien sous la forme d'une thèse ou d'un essai de maîtrise, ou encore sous la forme d'une recherche sociologique systématique. La liste de ces thèses et essais apparaît d'ailleurs à l'annexe 2.

La publication de ces cahiers, qui rapportent dans un langage précis mais simple les résultats de nos recherches et qui incorporent fortement la représentation visuelle, vient précisément répondre à l'intention initiale partagée au sein du GRAP: retourner aux groupes populaires concernés et à d'autres le produit des recherches, afin que celles-ci puissent être utiles immédiatement pour la formation des militants et pour l'élaboration d'une réflexion critique nécessaire sur l'action.

Vu les contraintes qui nous sont imposées dans le présent cadre, et à défaut de pouvoir élaborer davantage pour les deux autres secteurs, nous nous limiterons à scruter sommairement, sous la rubrique qui suit, comment s'est réalisée cette jonction entre les activités de recherche et l'action des groupes dans le seul secteur de la protection des consommateurs.

Par ailleurs, il nous faut tout de même prendre le temps de souligner l'importance de cette liaison d'échange pour chacune et pour l'ensemble des activités de recherche qui ont été menées au GRAP. Certaines de ces liaisons ont d'ailleurs produit des fruits dont l'amplitude ne pouvait guère être soupçonnée au départ.

Citons entre autres, à ce propos, l'importance du rapport d'échange qui fut établi à compter de 1977 entre les chercheurs du GRAP liés au secteur de la sécurité du revenu et le mouvement des assistés sociaux et des assistées sociales au Québec. 


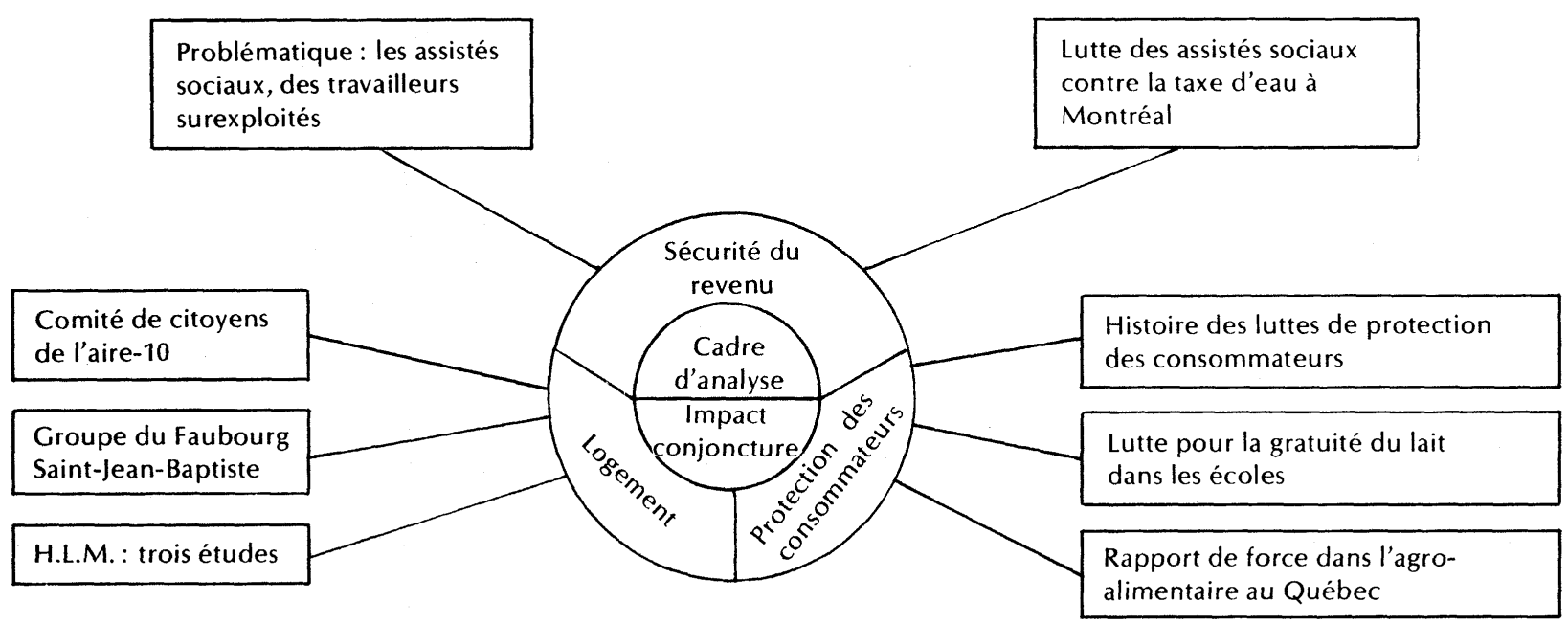

* Cette illustration tient compte uniquement des recherches publiées dans la série des Cahiers du GRAP. (Annexe 1.) 
En plus des bénéfices respectifs immédiats résultant de cette coopération entre les chercheurs du GRAP et les militants et militantes de cette organisation, ce rapport multiplia les effets périphériques à moyen terme. D'abord, en influant directement sur le développement de l'approche de conscientisation au niveau de la formation en organisation communautaire à l'École de service social de l'Université Laval. Puis, au sein du Regroupement des organisateurs et des organisatrices communautaires du Québec (ROCQ), en donnant un contenu concret à la perspective de conscientisation au niveau de sa propre pratique de formation et, à compter de mai 1982, au niveau de la forme de son manifeste et du nom même du Regroupement. C'est en effet à compter de cette date que le nom du ROCQ fut changé en celui de Collectif québécois de conscientisation. ${ }^{2}$

En contrepartie, les différentes organisations d'assistés sociaux et d'assistées sociales, tant le Front commun provincial qui entreprit de se constituer à compter de 1977 que certaines associations locales ou régionales de défense des droits sociaux (A.D.D.S.), ont pu largement tirer profit des recherches menées par le GRAP dans le secteur particulier de la sécurité du revenu (voir les cahiers 2 et 8 mentionnés à l'annexe 1). Elles ont pu également tirer grand avantage des outils du cadre d'analyse du GRAP, tant à des fins de formation militante qu'au niveau d'une réflexion critique et stratégique constante sur l'action. Ces mêmes outils, principalement élaborés par Gérald Doré, sont présentés dans les cahiers 3 et 4, qui ont été publiés en 1981. Enfin, cette liaison dynamique a également joué dans le sens d'une ouverture des portes du ROCQ à un certain nombre de militantes assistées sociales et de militantes et militants alliés leur étant associés. Ce dernier fait n'a pas été étranger, de toute évidence, aux importants changements intervenus au sein du ROCQ à compter de 1978, et dont nous avons brièvement fait mention auparavant.

Comment ne pas se sentir quelque peu mal à l'aise de devoir rendre compte de cette histoire riche et complexe d'une façon si sommaire!

\section{Sur cette liaison entre la recherche et l'action dans le secteur de la protection des consommateurs}

C'est à l'automne de 1977 que le collègue Marc Roland et moi entreprenions de débroussailler le terrain de notre secteur de recherche : la protection des consommateurs. Cette responsabilité, nous la partagerons d'ailleurs intensément jusqu'au terme des opérations. 
Notre premier travail fut alors de réfléchir et de prendre position au sein du GRAP sur le sens et les exigences d'une liaison organique véritable entre la démarche de recherche et l'action des groupes populaires concernés dans notre secteur. L'idée de base était la suivante : établir au préalable une entente claire et explicite entre nous comme chercheurs et les militants sur le terrain, afin que se développe un rapport d'échange véritablement égalitaire entre les deux parties concernées et, surtout, que la recherche soit prioritairement au service de l'action et des luttes, plutôt que l'inverse. Idéalistes, nous tenions fermement à ce principe, sans trop savoir comment y donner forme, mais nous y tenions comme à une norme morale. Nous le vivions comme un refus de spolier l'action pour satisfaire les intérêts de la recherche. C'est de cette base de départ que nous avons amorcé l'aventure, sans savoir où elle allait effectivement nous conduire.

Déjà, depuis 1975, j'assumais la délégation du Syndicat des professeurs de l'Université Laval (SPUL) auprès de l'Association coopérative d'économie familiale de Québec (ACEF) et, depuis là, auprès de la Fédération des associations coopératives d'économie familiale au Québec (FACEF).

Marc Roland, pour sa part, était également en liaison avec les permanents et bon nombre de militants et de militantes de l'ACEF de Québec, étant donné son implication auprès de nombreux groupes populaires à Québec depuis plusieurs années.

De plus, la plupart d'entre nous, nous nous retrouvions comme militants de la première heure au sein d'un nouveau parti politique municipal, le Rassemblement populaire. Celui-ci avait émergé officiellement en janvier 1977, dans le sillon des luttes populaires menées au cours des dix années précédentes sur le front de l'habitat et de la rénovation urbaine à Québec. II faudra bien un jour écrire un morceau de cette page de notre histoire de vie!

Dans l'immédiat, retenons simplement qu'en 1977, au moment où nous avons amorcé la réalisation de notre portion de recherche au GRAP, Pierre Racicot cumulait en même temps les fonctions de gérant de l'ACEF de Québec et de président du Rassemblement populaire. Pour ma part, à l'automne de 1977, j'étais également candidat du Rassemblement populaire dans mon district, pour l'élection tenue en novembre. Et Marc Roland était membre du Comité de coordination du Parti, dont il avait d'ailleurs été l'un des pionniers avec sept autres militants, dont Gérald Doré et Pierre Racicot, entre autres. Marc sera également candidat du Parti dans son district à l'élection municipale de 1981 et à celle de 1985. Gérald fera de même en 1981. Et, après que Pierre Racicot aura quitté la présidence du Rassemblement populaire 
en 1983 avec son équipe, c'est Marc et moi qui en assumerons la relève à la présidence et à la vice-présidence jusqu'à la fin de 1984 .

Si j'ai jugé bon de faire ce bref détour historique, c'est pour montrer qu'une recherche-action à caractère militant ne peut pas émerger et prendre forme par hasard, pas plus qu'elle ne peut résulter du simple désir ou de l'intention de chercheurs en dehors de l'action. Cela suppose plutôt qu'ils sont déjà présents, comme acteurs et militants, au cour de la praxis des luttes où ils choisissent d'inscrire leur contribution propre.

Dans notre secteur de recherche, cette liaison organique, définie au départ comme l'établissement d'un rapport d'échange égalitaire entre les chercheurs et les militants, rapport où la recherche serait prioritairement au service de l'action, prit une forme imprévue dont je vais maintenant tenter de reconstituer l'essentiel de la trame historique.

C'est au printemps de 1977 qu'il faut faire remonter le début de cette petite histoire. À l'instar des autres groupes du mouvement populaire et du mouvement syndical au Québec, les ACEF sont alors traversées de part en part par les grands débats d'orientation, débats auxquels la pâte militante marxiste-léniniste contribuera à donner des allures doctrinales et des orientations sectaires, qui seront une source de profonde division et de démobilisation.

Mon intention ici n'est pas de discuter de certaines utilités ou encore de l'impact négatif de cette offensive menée par les marxistes-léninistes auprès des groupes populaires et des syndicats, entre 1974 et 1978 principalement. Je veux simplement retenir le fait que cette forme de gauchisme, marquée du sceau de la foi religieuse, et principalement portée par des intellectuels capables d'un discours théorique articulé, mais aussi parfaitement débilitant parce que sans correspondance avec les conditions et les possibilités de la réalité du moment, a joué au sein du mouvement des ACEF comme ailleurs où elle est parvenue à prendre racine, dans le sens d'une dislocation des forces et d'une démobilisation des militants et militantes, en particulier ceux et celles provenant directement des classes populaires.

Pour les ACEF, cela a signifié la scission de leur mouvement provincial, la FACEF, lors des assises du grand Congrès d'orientation tenu au printemps de 1977 à Sherbrooke. J'y étais en tant que participant au débat comme délégué de l'ACEF de Québec.

Devant les nouvelles orientations du mouvement décidées par le Congrès, antérieurement bien cuisinées par l'équipe des permanents de la Fédération et celle de l'imposante ACEF de Montréal, il n'y avait plus guère d'alternatives. Rentrer dans le rang et se conformer au nouveau "centralisme démocratique" du mouvement, héritage des 
marxistes-léninistes d'extrême-gauche, ou bien être dissident et, par conséquent, se retirer. C'est effectivement ce que fera l'ACEF de Québec avec trois autres associations locales.

Dès le lendemain de ce Congrès, le projet de mettre sur pied une nouvelle organisation rassemblant les ACEF dissidentes commencera à germer. Elle sera finalement établie à l'automne de 1977, sous le nom de Fédération nationale des associations de consommateurs et consommatrices du Québec (FNACQ).

À cette manche importante de l'histoire du mouvement des ACEF, j'ai été très étroitement lié comme délégué du SPUL auprès de l'association de Québec et comme militant actif au sein de celle-ci. Au moment d'entreprendre les travaux dans notre secteur, à l'automne 1977, c'est dans le cadre de cette conjoncture de crise du mouvement des consommateurs au Québec que nous devions nous situer, Marc Roland et moi, comme chercheurs du GRAP.

C'est donc au cœur même de l'enjeu de la survie des ACEF dissidentes, avec lesquelles nous avions partie liée par le biais de ma propre pratique militante, que nous avons vu dès lors se dessiner les premiers objets de recherche qui devaient nous mobiliser, si nous voulions contribuer à l'action.

Car survivre signifiait tracer des orientations pour l'organisation et pour sa pratique de service et de lutte. Et cela commandait à la fois une réflexion critique sur notre histoire collective et sur les perspectives possibles et souhaitables de notre développement.

C'est également dans le creuset de cette nouvelle praxis que devait progresser la formation technique et politique des permanents et des militants. Les durs combats idéologiques menés contre les offensives marxistes-léninistes au cours des dernières années nous avaient révélé avec force l'urgent besoin d'une telle formation politique. Et c'est par la réorganisation des forces du mouvement et dans l'action que celle-ci devait prendre place et se réaliser. Dans ce contexte, le terme de praxis référait à une dialectique complexe définie sous la forme d'un triple rapport :

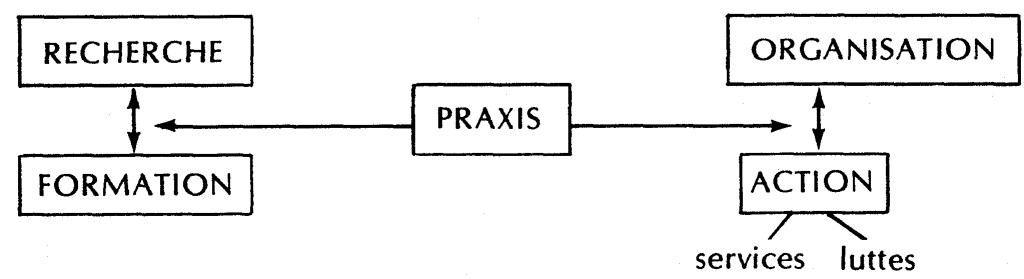


Pour répondre aux exigences de cette conjoncture au sein de I'ACEF de Québec et de la nouvelle FNACQ, Marc Roland et moi entreprenions dès lors deux séries de travaux, vus comme étant de nature à contribuer au développement de cette praxis au sein du mouvement. La première portait sur une analyse historique des luttes menées par le mouvement de protection des consommateurs au Québec entre 1962 et 1978. Cette recherche sociologique, inspirée du cadre d'analyse du GRAP " bilan d'impact», donna lieu à la production d'un rapport élaboré, dont le résumé prit la forme du premier cahier publié par le GRAP en 1980.

C'est d'ailleurs à partir du dessein de ce premier cahier, dont nous pensions qu'il pouvait bien concrétiser notre intention de vulgariser les résultats de nos recherches aux fins d'une démarche d'éducation populaire et de formation militante au sein des groupes, que l'ensemble des autres cahiers seront produits.

Parallèlement à cette analyse de l'histoire du mouvement et de ses luttes, nous entreprenions une importante réflexion à caractère stratégique sur la conjoncture sociale et les défis du mouvement sur le front des luttes de protection des consommateurs au Québec, à l'aube des années 1980. Les résultats de cette réflexion se sont cristallisés dans un ensemble de considérations d'ordre stratégique, formulées autour de quatre grands thèmes, et comportant l'élaboration de toute une panoplie "d'hypothèses d'action pour les ACEF-FNACQ".

Le matériel de cette réflexion critique sur l'action fut livré aux permanents et aux militants du mouvement, au début de 1980, encore une fois sous la forme de quatre petits cahiers de vulgarisation, dont les thèmes sont : ${ }^{3}$ Enjeux et front de lutte, 19 pages; Mobilisation, conscientisation et direction politique, 23 pages; Sur les rapports avec l'État et les compagnies, 24 pages; et Conditions... d'une stratégie de développement et de lutte, 19 pages.

À compter de 1980, cette série de cinq cahiers de recherche fournit les matériaux nécessaires aux permanents et aux militants du mouvement pour initier et nourrir toute une démarche de formation et de réflexion sur l'action. À certaines occasions, cette démarche prendra la forme de sessions intensives d'une journée, auxquelles nous serons directement associés, à titre de chercheurs, militants et personnes ressources.

C'est dans la foulée du même cheminement, en cours depuis l'automne 1977, qu'allaient encore une fois se dessiner les questions nous conduisant vers la définition d'autres objets de recherche susceptibles de mobiliser nos énergies. 
En effet, depuis 1972-1973 le mouvement des ACEF avait entrepris de lutter contre les hausses du prix du lait. À compter de 1975, l'enjeu de cette lutte s'était centré autour de la gratuité du lait dans les écoles. Après la scission avec la FACEF, au printemps de 1977, et ce jusqu'à l'obtention effective de la mesure par une décision du gouvernement du Parti québécois, en 1978, l'ACEF de Québec sera la seule à poursuivre cette lutte.

C'est principalement pour pouvoir mieux tirer les leçons de cette lutte que Marc Roland entreprendra d'en faire le bilan, à l'aide du cadre d'analyse du GRAP. Les résultats de cette recherche seront par la suite résumés et publiés dans le sixième cahier du GRAP. Le contenu de ce cahier sera aussi l'objet d'un débat entre les chercheurs, les permanents et les militants de l'ACEF de Québec. Mais sur les conclusions de cette étude, lesquelles mettent en doute l'importance déterminante de l'action de l'ACEF par rapport à l'issue positive de cette lutte, le débat ne permettra pas de parvenir à une entente!

Ces premières offensives, menées par le mouvement des ACEF sur la question du prix du lait et de sa distribution gratuite dans les écoles, avaient graduellement contribué à mettre à jour toute l'importance des dépenses alimentaires dans le budget hebdomadaire des familles, en particulier celles à faible revenu.

À compter de 1978, et suite au cheminement de réflexion collective auquel nous avons fait référence ci-avant, le front de l'alimentation tendra à devenir un enjeu prioritaire de lutte sociale et économique au sein de l'ACEF de Québec et de la FNACQ. Mais pour parvenir à bien identifier les cibles visées, encore fallait-il bien connaître les configurations, les acteurs et la dynamique interne et externe propres à cet imposant espace. Ce qui n'était pas le cas. C'est donc à cette tâche que je consacrerai mes énergies au cours des années qui suivront. Pour ce faire, un vaste chantier de recherche sera défini et trois études seront produites, portant sur les objets suivants : le développement de l'agroalimentaire au Québec, du début de la colonie à 1980; la conjoncture politique (i.e. le rapport de force) dans l'ensemble de l'espace de l'agro-alimentaire au Québec en 1980; la conjoncture politique actuelle au sein de la filière laitière.

La première et la troisième de ces études, bien qu'achevées, ne seront pas publiées faute de ressources. Quant à la seconde, la plus importante des trois d'ailleurs, elle formera le matériau principal utilisé pour les fins de ma thèse de doctorat en sociologie. ${ }^{4} \mathrm{Et}$ les conclusions de celle-ci constitueront le contenu du cahier 7 du GRAP, publié en 1982. 
Ce dernier cahier retournera sur le terrain à compter de cette date et il sera mis à contribution par le Comité agro-alimentaire de l'ACEF de Québec et de la FNACQ pour guider le développement de cette lutte principale menée désormais sur l'ensemble de ce front particulier des conditions de vie.

Il faut également savoir que, tout au long de l'histoire de notre recherche, réalisée en liaison avec la pratique de l'ACEF de Québec et de la FNACQ, les occasions de déployer notre implication militante au sein du mouvement se multiplieront. Retenons à cet égard, entre autres, ma participation aux différents dossiers de l'ACEF de Québec, comme membre du Conseil d'administration entre 1976 et 1978; notre association, Marc Roland et moi, aux différentes étapes de la lutte menée en rapport avec l'adoption de la Loi 72 sur la protection des consommateurs au Québec, à l'automne 1978; ma participation aux audiences de la Régie des marchés agricoles de juin 1979 sur la question du prix du lait, auprès du représentant de la FNACQ; ma participation comme conseiller de la FNACQ au mini-sommet socioéconomique du Québec sur les produits laitiers, tenu en mars 1980 à Rimouski ; notre contribution ponctuelle aux activités du Comité agroalimentaire de I'ACEF de Québec, mis sur pied en 1979-1980; et, finalement, ma participation comme acteur privilégié (i.e. "expert") à une émission télévisée de Radio-Québec, en janvier 1985, sur la question des prix élevés des aliments, émission de "Action débat" ayant mis en confrontation directe les groupes de consommateurs et différents groupes populaires, aux positions desquels je me suis associé, et les représentants des quatre grandes chaînes du commerce de l'alimentation, présentes pour la première fois à une émission de ce genre au Québec.

Quelles leçons peut-on maintenant tirer de cette expérience vécue avec tant d'intensité au cours de toutes ces années?

\section{Quelques considérations en marge de notre expérience de recherche-action}

Il aurait certes été intéressant de discuter, ici, des caractéristiques formelles de la recherche-action à caractère militant, nous inspirant de notre expérience et la situant dans le cadre plus strict d'une typologie de la recherche sociologique.

Cette opération, que nous avons d'ailleurs menée en 1983 dans le cadre d'un séminaire de maîtrise en mouvements populaires à l'École de service social de l'Université Laval et tenu avec Colette Humbert, est 
laborieuse et de nature plus académique; elle exige des élaborations qui ne sont guère compatibles avec la longueur du présent texte.

Voilà pourquoi nous nous limitons à référer le lecteur ou la lectrice à l'annexe 3, où il sera possible de repérer les liens de parenté de notre propre démarche avec deux des cinq formes de recherche définies dans le cadre de la typologie qui y apparait, soit la rechercheaction et la recherche militante. ${ }^{5}$

En contrepartie, il nous semble utile de tirer certaines leçons de notre expérience, et de livrer simplement ces réflexions personnelles et quelques considérations jugées plus fondamentales à ceux et celles qui pourraient se sentir attirés par l'idée de construire puis de vivre pareille aventure.

La première de ces considérations concerne la mise en place des ressources nécessaires à la production de la recherche. À cet égard, il ne nous apparaît guère réaliste de croire que la recherche militante puisse strictement résulter du déploiement d'énergies volontaires, surtout lorsque les objets de recherche en cause commandent une importante élaboration.

Dans ce contexte, trouver un financement suffisant pour assurer la réalisation des opérations jusqu'à terme devient une question délicate. Cela suppose, nous semble-t-il, deux conditions. D'une part, les qualités théoriques et méthodologiques du projet soumis aux bailleurs de fonds doivent se conformer aux grands canons de la science académique, car les personnes qui disposeront du projet en sont le produit fidèle. D'autre part, on n'insistera jamais trop sur l'importance stratégique de faire certains compromis conceptuels dans la formulation du projet soumis, sans toutefois trahir le sens même de la démarche entreprise.

À cet égard, il importe d'être bien conscient du fait que le vocabulaire de la gauche, surtout lorsqu'il répercute directement I'héritage du matérialisme historique et du matérialisme dialectique, ne souffre guère d'un excès de popularité dans les enceintes institutionnelles qui financent la recherche. Sous ce chapitre, il faut donc être tout à la fois cohérent et rusé.

Un autre défi de la recherche-action à caractère militant, c'est de durer... pour parvenir à terme, et réaliser l'ensemble des objectifs visés. Cette question, fort pratique, n'est toutefois pas simple. Elle pose bien sûr le problème d'un financement de départ adéquat, mais il y a également les problèmes qui peuvent découler de l'envergure du chantier et de sa durée. Et oui, le temps !... qui ne joue pas toujours en notre faveur. Car, après un certain nombre d'années, les ressources financières deviennent rares, sinon épuisées, et les membres de 
l'équipe de recherche, pour survivre ou pour d'autres raisons, doivent commencer d'inventorier de nouveaux horizons de travail et de vie. C'est là un défi que nous avons rencontré au GRAP à compter de 1981-1982. À la fin de 1985, les dernières productions prévues tardent toujours à prendre forme et les ressources humaines disponibles sont devenues plutôt rares. Seuls continuent d'être dans le décor le pionnier de cette recherche, Gérald Doré, et nous-même.

II faut également savoir que depuis la fin de la subvention, en 1981, la publication des nouveaux cahiers produits a dû et doit toujours s'autofinancer au moyen des recettes de vente des cahiers parus antérieurement. Ce qui n'est pas une mince affaire.

La troisième considération dont il est important de faire état concerne la nature des rapports de production de la recherche-action à caractère militant. Cette question est fondamentale; elle se situe au cour même du sens de ce type de démarche et d'aventure. Cette question interroge la forme du rapport de travail qui s'établit entre les membres de l'équipe de recherche, d'une part et, d'autre part, entre celle-ci et la pratique des groupes sur le terrain, qui sont concernés et visés par ses produits.

Cette question est fondamentale car elle repose sur une option idéologique et politique, un projet de société qui anime les membres du groupe porteur de l'expérience. Ce projet se caractérise par une volonté de changement radical, où les rapports d'oppression qui structurent présentement la réalité sociale devraient être remplacés par de nouveaux rapports coopératifs et d'échange égalitaire.

Or, lorsque nous sommes, comme produit social, des INTELLECTUELS à notre image, le développement de cette forme de rapports coopératifs et d'échange plus égalitaire est loin de constituer un acquis de départ; bien au contraire! II peut même arriver, qu'au terme de l'aventure, nos efforts à cet égard ne fassent que commencer. Cela pose tout le problème de l'intellectuel, comme produit social structuré sous une forme mythique (i.e. celui qui détient la connaissance); mythe dont sont généralement victimes non seulement les citoyens des classes populaires mais les intellectuels eux-mêmes. "Sacrés z'intellectuels, va...!".

Au sein du GRAP, cette réalité a joué principalement dans le sens d'une grande difficulté à parvenir à établir une atmosphère de travail, d'échange et de débat et des attitudes propres à favoriser le développement de relations coopératives et égalitaires. Ce qui n'a pas été sans faire émerger de grandes tensions entre certains d'entre nous, à quelques occasions. Quoi qu'il en soit, cela fait maintenant partie de la petite histoire de notre famille. 
C'est d'ailleurs peut-être entre les membres du GRAP et les différents groupes populaires concernés par la recherche que cette liaison d'échange et de coopération fut la plus intéressante et la plus productive. Sous les rubriques qui précèdent, nous avons tenté d'en témoigner en l'illustrant de quelques exemples vécus au sein de notre groupe.

Sous ce chapitre, les exemples dont il est fait mention permettent de retenir trois éléments principaux comme constituants nécessaires à toute recherche-action qui veut s'élaborer dans une perspective militante.

D'abord, il est primordial que les chercheurs soient au départ directement liés à la pratique d'action qu'ils aspirent servir par la réalisation de leurs recherches. Comme nous l'avons clairement démontré au début de la rubrique qui précède, une recherche militante ne peut prendre forme ni par le hasard, ni par la simple volonté des chercheurs pour qu'il en soit ainsi. Elle naît de l'action et elle doit émerger de ceux et celles qui la font.

Cette considération nous amène à établir un deuxième constat, qui se veut être un principe. Contrairement à la recherche sociologique classique, ou encore de type académique, la recherche-action de type militant doit poser son regard sur les questions centrales qui émergent des défis que confronte le développement de la pratique d'action, à un moment précis de son histoire. Ce sont ces mêmes défis qui déterminent les objets de la recherche. C'est à cette seule fin et dans ce seul cadre que les chercheurs et leurs recherches peuvent constituer un apport à la praxis concrète des groupes concernés.

Le troisième élément concerne la forme des produits de la recherche. À cet égard, notre expérience vécue au sein du GRAP a démontré clairement, s'il était encore nécessaire de nous en convaincre, qu'il était strictement inutile de retourner auprès des groupes concernés, de leurs permanents et de leurs militants, les rapports élaborés de nos recherches.

Est-il besoin de rappeler à ce propos que l'objectif central de toute recherche-action à caractère militant est de constituer le moyen privilégié d'une réflexion critique sur l'action, articulée à une démarche de formation de tous ceux qui la portent: les permanents et les militants.

Cela comporte des exigences précises sur le plan des outils et de la démarche pédagogiques, qui ne trouvent guère satisfaction dans le cadre classique des rapports de recherche volumineux, livrés sous une forme scholastique, sans plus d'égards ni d'inquiétudes quant aux suites. 
C'est ce qui nous a incités à mettre au point la formule des cahiers du GRAP. Ceux-ci parviennent à rendre compte des résultats essentiels des recherches; ils sont peu volumineux; le langage est simple et l'illustration y est abondante.

Cette forme de production est exigeante puisqu'elle constitue un tout autre produit que celui des rapports de recherche. Elle est par ailleurs essentielle, si nous as pirons à ce que les groupes parviennent à s'approprier véritablement du produit de la recherche comme telle. Comme preuve de l'intérêt porté à cette forme de document, mentionnons simplement que les onze cahiers publiés par le GRAP jusqu'à ce jour ont connu un tirage de 6500 exemplaires, dont la plus grande partie est maintenant écoulée.

Mais l'histoire ne se termine pas là. Il importe que les chercheurs poursuivent leur implication militante au sein du groupe afin que ce matériel, une fois vulgarisé, s'incorpore graduellement à la praxis de formation, d'organisation et d'action de celui-ci. Et cela peut signifier, non pas quelques semaines ou quelques mois, mais des années! C'est là qu'il faut apercevoir l'exigence fondamentale d'une rechercheaction de type militant. Elle est une option qui constitue également un choix de vie.

\section{Pour une sociologie militante}

"La science n'est jamais neutre [écrit notre collègue Andrée Fortin], non seulement à cause des différentes applications qui peuvent être faites de ses résultats, mais aussi à cause des problèmes qu'elle se pose - ou non - et des méthodes qu'elle utilise pour les résoudre.» ${ }^{6}$

En ce qui nous concerne, les recherches que nous avons menées au sein du GRAP, comme l'ensemble des autres recherches réalisées au sein de celui-ci à compter de 1975-1976, ont voulu prendre ancrage au cœur même des défis actuels des pratiques populaires de lutte, déployées sur le front des conditions de vie.

Le procès d'élaboration de ces recherches, malgré certaines limites, a voulu se fonder sur l'établissement d'un rapport d'échange constant entre les artisans de cette double pratique : chercheurs et militants.

Et le produit des recherches retourné aux groupes, soit les cahiers de vulgarisation, a dû faire l'objet d'une construction spécifique, empruntant la forme de certaines exigences propres à une démarche d'éducation populaire et de formation militante au sein des groupes.

Au terme de ce long périple, nous ne pouvons omettre de prendre position par rapport aux conditions et aux perspectives de la pratique 
sociologique, dans la conjoncture actuelle. En effet, dans cette conjoncture où les défis de la praxis sociale libératrice sont si imposants, celle-ci est plus souvent qu'autrement confrontée à sa propre impuissance, à son propre vide, plutôt qu'elle ne parvient à constituer un apport significatif, à titre d'éclairage sur les grands enjeux par rapport auxquels cette praxis sociale libératrice doit présentement chercher à prendre forme.

Wright C. Mills écrivait, en 1967, dans son livre "L'imagination sociologique":

"Ma conception de la sociologie n'est pas dans le vent. Elle condamne la science des techniques bureaucratiques, qui inhibent la recherche par des prétentions "méthodologiques", l'alourdissent de conceptions confuses, la galvaudent sous les problèmes mineurs coupés des enjeux collectifs. [...]

"Ces inhibitions, ces obscurités, ces banalités ont plongé les études dans la crise, sans en suggérer l'issue le moins du monde.» ${ }^{7}$

D'emblée, nous partageons ce point de vue critique. Nous savons trop bien qu'au cours des vingt années qui ont suivi l'établissement de ce constat de Mills, la pratique sociologique, du moins la portion principale de celle-ci, n'est guère parvenue à trouver sa voie, à construire son propre projet.

Voilà pourquoi nous avons cherché, au sein du GRAP, à inventorier de nouvelles pistes pouvant conduire au sens d'une certaine utilité sociale, propre à notre contribution et à notre métier de sociologue.

Cette recherche de perspectives nouvelles s'est finalement cristallisée dans une démarche de recherche-action de type militant, dont le caractère et la validité scientifiques ont été souventes fois contestés par ceux qui ont la prétention d'être les dépositaires de la seule vraie science. Comme s'il pouvait exister deux sortes de science, une vraie et une fausse, dépendant de la distance au réel de la part de ceux qui l'élaborent.

À cet égard, nous voulons nous ranger parmi les dissidents d'une sociologie académique qui identifie souvent la scientificité de la connaissance au formalisme de sa démarche et au caractère ésotérique de ses produits. De même, nous sommes dissidents du grand courant de la sociologie aseptique, "neutre", "objective», et dont les prétentions scientifiques sont strictement liées au fait qu'elle parvient à s'élaborer en retrait du réel, comme au-dessus de la mêlée.

Notre parti pris c'est celui d'une sociologie militante, d'une sociologie critique profondément inscrite dans la pratique sociale et historique, consciente de ses contradictions et de ses limites mais aussi 
du fait qu'elle constitue essentiellement une "science de/dans la société". ${ }^{8}$ Cette sociologie n'a rien de commun avec le discours partisan déguisé sous couvert de la science, et pas davantage avec l'écrit pseudo-scientifique réservé aux initiés.

La sociologie militante, c'est celle qui tend à articuler de manière dialectique et dans une même pratique, le procès de connaissance de la réalité et l'acte de sa transformation; et ce, sur une base critique qui veut rendre compte de cette réalité avec justesse et objectivité, sans pour autant chercher à "compliquer inutilement les choses". ${ }^{9}$ Cette sociologie prend nécessairement le parti d'être un outil parmi d'autres au service du projet libérateur contre toutes les formes d'oppression et, par conséquent, contre la structure et les rapports sociaux qui en assurent la reproduction.

Ce choix, finalement, c'est celui qui tend à réaliser le projet de Gramsci de l'intellectuel organique, de l'intellectuel qui cherche, malgré les limites qui lui sont propres, à établir alliance et soudure avec les pratiques libératrices des masses populaires, de même qu'avec les luttes menées par tous ceux et celles qui subissent l'oppression.

\section{Notes et références}

1 Doré, G., V. Faleiros et D. Fortin, Impact des associations volontaires de politique sociale, projet soumis au Comité de la recherche socio-économique du M.A.S., Québec, Laboratoire de recherches en service social, décembre 1975, p. 1.

2 Pour compléter cette brève reconstitution historique, il faut lire : AMPLEMAN, G., G. Doré, L. Gaudreau, C. Larose, L. Leboeuf et D. Ventelou, Pratiques de conscientisation. Expériences d'éducation populaire au Québec, Montréal, Nouvelle Optique, 1983 ; en particulier le chapitre 4, pp. 101-129.

${ }^{3} \mathrm{Vu}$ leur nature, ces quatre cahiers ne font pas partie des publications du GRAP. Ils constituent des productions hors-série et autonomes, dont il est possible d'obtenir copie en s'adressant à l'ACEF de Québec ou à la FNACQ.

4 FORTIN, D., Sur l'agro-alimentaire au Québec. Conjoncture politique actuelle, thèse de doctorat en sociologie, Faculté des sciences sociales, Université Laval, 1981, 667p.

${ }^{5}$ Sur ce sujet, voir également: Colette Humbert et Jean Merlo, L'enquête conscientisante. Problèmes et méthodes, Paris, L'Harmattan, 1978; pp. 8-11 principalement.

6 Fortin, Andrée, "La sociologie de/dans la société», Sociologie et sociétés, vol. XII, $\mathrm{n}^{\circ} 2,1980$ : 93-94. 
7 Mills, Wright C., L'imagination sociologique, Paris, Maspero, 1967, p. 24. ("Textes à l'appui».)

${ }^{8}$ FORTIN, Andrée, op. cit., p. 75.

9 Id., p. 93.

\section{ANNEXE 1 \\ Cahiers du GRAP déjà parus *}

Denis Fortin et Marc Roland, Histoire des luttes de protection des consommateurs, 1962-1978, cahier 1, 1980, 26p.

Vicente Faleiros, Marc Roland, Denise Ventelou, et l'O.P.D.S.-Mercier, Les assistés sociaux : des travailleurs surexploités, cahier 2, 1981, 31p.

Gérald Doré, Pour faire le bilan de nos luttes : étude de l'impact, cahier 3, 1981, $29 p$.

Gérald Doré, Le jeu des forces sociales dans nos luttes: analyse de la conjoncture, cahier 4, 1981, 34p.

Gérald Doré et Denis Plamondon, Gagner ou perdre: deux luttes sur le logement à Québec, cahier 5, 1981, 33p.

Denis Fortin et Marc Roland, La gratuité du lait dans les écoles: une lutte de consommateurs?, cahier 6, 1981, 22p.

Denis FORTIN (en collaboration avec Marc ROLAND), L'agro-alimentaire au Québec : un rapport de force, cahier 7, 1982, 55p.

Denise Ventelou (en collaboration avec l'O.P.D.S.-Mercier), La lutte des assistés sociaux de Montréal contre la taxe d'eau: d'une autoréduction à l'éveil d'une conscience politique, cahier 8, 1983, 38p.

Pierre FourNIER, Les locataires de H.L.M. face à leurs conditions de vie : le vidéo comme outil de conscientisation, cahier 9, 1983, 37p.

Michelle Arcand, Pierre Fournier et Denis Plamondon, Vivre en H.L.M. : les conditions de vie dans les logements municipaux subventionnés, cahier 10, 1983, 36p.

Carole RoBerge, Une lutte pour des services dans un H.L.M.: le piège des programmes de création d'emplois, cahier 11, 1984, 24p.

* Adresse: GRAP, École de service social, Université Laval, Québec (QC) G1K 7P4. 


\section{ANNEXE 2 \\ Principales autres productions liées à la recherche du GRAP}

Deux thèses de doctorat, quatre thèses de maîtrise et trois essais de 12 crédits ont été complétés dans le cadre de cette recherche ou lui ont fait des emprunts théoriques et empiriques:

Gérald Doré, La politique sociale comme pratique de classes, thèse de doctorat en sociologie, Université Laval, 1978, 514p.

Denis ForTIN, Sur l'agro-alimentaire au Québec, thèse de doctorat en sociologie, Université Laval, 1981, 667p.

Michelle ARCAND, La participation des locataires à la gestion des H.L.M. de Québec, de 1971 à 1978, thèse de maîtrise en service social, Université Laval, 1981, 211p.

Jocelyne BARnABÉ, L'échec scolaire et la classe sociale chez les élèves de secondaire I à la Commission des Écoles catholiques de Québec en 19761977, thèse de maîtrise en service social, Université Laval, 1978, 207p.

Carole Roberge, Lutte des locataires des Appartements Saint-Pie X pour l'implantation de services dans leur complexe d'habitation H.L.M., 1971-1977, thèse de maîtrise en service social, Université Laval, 1981, 337p.

Denise Ventelou, La lutte des assistés sociaux de Montréal contre la taxe d'eau, thèse de maîtrise en service social, Université Laval, 1981, 207p.

Pierre Fournier, Le vidéo comme outil de conscientisation. Une expérience avec des locataires de logements municipaux, essai de 12 crédits en service social, École de service social, Université Laval, 1981, 143p.

Christian Jouın, La situation de classe du Rassemblement populaire à Québec en 1978, essai de 12 crédits en service social, École de service social, Université Laval, 1981, 115p.

Denis Plamondon, La lutte du Groupe du Faubourg contre la tour d'Aiguillon, essai de 12 crédits en service social, École de service social, Université Laval, 1978, 274p. 
ANNEXE 3

La recherche sociologique : typologie de Colette Humbert*

\begin{tabular}{|c|c|c|c|}
\hline $\begin{array}{l}\text { Type } \\
\text { d'enquête }\end{array}$ & Commanditaires & $\begin{array}{l}\text { Milieu / Popula- } \\
\text { tion concernés }\end{array}$ & $\begin{array}{l}\text { Sujets de la } \\
\text { recherche }\end{array}$ \\
\hline Informative & $\begin{array}{l}\text {-Organisations ou } \\
\text { entreprises (pro- } \\
\text { blème d'échelle } \\
\text { et de hiérarchie } \\
\text { sociale) }\end{array}$ & $\begin{array}{l}\text {-Toute population } \\
\text { (par secteurs ver- } \\
\text { ticaux intégrés) }\end{array}$ & $\begin{array}{l}\text {-Le chercheur ou } \\
\text { l'équipe de re- } \\
\text { cherche profes- } \\
\text { sionnelle }\end{array}$ \\
\hline $\begin{array}{l}\text { Participa- } \\
\text { tive }\end{array}$ & $\begin{array}{l}\text {-Organisations ou } \\
\text { entreprises }\end{array}$ & $\begin{array}{l}\text {-Populations plus } \\
\text { spécifiques (per- } \\
\text { sonnel d'une en- } \\
\text { treprise, d'un quar- } \\
\text { tier, etc.) }\end{array}$ & $\begin{array}{l}\text {-Le(s) chercheur(s) } \\
\text { plus des repré- } \\
\text { sentants de la po- } \\
\text { pulation, selon } \\
\text { le modèle social } \\
\text { donné }\end{array}$ \\
\hline $\begin{array}{l}\text { Recherche- } \\
\text { action }\end{array}$ & $\begin{array}{l}\text {-Organisations } \\
\text {-Institutions inter- } \\
\text { médiaires (univer- } \\
\text { sités) } \\
\text {-Organisations com- } \\
\text { munautaires }\end{array}$ & $\begin{array}{l}\text {-Populations-cibles } \\
\text { (susceptibles de } \\
\text { se mobiliser sur } \\
\text { un besoin) } \\
\text {-Groupes ruraux/ } \\
\text { urbains }\end{array}$ & $\begin{array}{l}\text {-Collectif de cher- } \\
\text { cheurs plus des } \\
\text { associations ex- } \\
\text { primant le dyna- } \\
\text { misme de la po- } \\
\text { pulation }\end{array}$ \\
\hline $\begin{array}{l}\text { Conscienti- } \\
\text { sante }\end{array}$ & $\begin{array}{l}\text {-Groupes militants } \\
\text { d'une population } \\
\text { à la base }\end{array}$ & $\begin{array}{l}\text {-Milieux populaires/ } \\
\text { classes populaires } \\
\text { dans une situation } \\
\text { d'oppression (éco- } \\
\text { nomique, politique } \\
\text { ou culturelle) }\end{array}$ & $\begin{array}{l}\text {-Groupes militants } \\
\text { au sein de la po- } \\
\text { pulation } \\
\text {-Population } \\
\text {-Animateurs soli- } \\
\text { daires des groupes } \\
\text { militants }\end{array}$ \\
\hline $\begin{array}{l}\text { Recherche } \\
\text { militante }\end{array}$ & $\begin{array}{l}\text {-Enquête auto-dé- } \\
\text { cidée par un petit } \\
\text { groupe militant }\end{array}$ & $\begin{array}{l}\text {-Groupes sociaux } \\
\text { opprimés recon- } \\
\text { nus par les mili- } \\
\text { tants comme "por- } \\
\text { teurs d'espoir et } \\
\text { de besoin de chan- } \\
\text { gement" }\end{array}$ & $\begin{array}{l}\text { - Les chercheurs- } \\
\text { militants institués } \\
\text { avant-garde du } \\
\text { groupe de popu- } \\
\text { lation }\end{array}$ \\
\hline
\end{tabular}

* Cette typologie a été présentée par Colette Humbert lors d'un séminaire de maîtrise en mouvements populaires, tenu en avril 1983 à l'École de service social de l'Université Laval. 
ANNEXE 3 (suite)

\begin{tabular}{|c|c|c|}
\hline $\begin{array}{l}\text { Objets de la } \\
\text { recherche }\end{array}$ & $\begin{array}{c}\text { Acteurs par } \\
\text { priorités d'intérêts }\end{array}$ & $\begin{array}{l}\text { Démarche } \\
\text { méthodologique }\end{array}$ \\
\hline $\begin{array}{l}\text {-Les caractéristiques mul- } \\
\text { tiples d'une population }\end{array}$ & $\begin{array}{l}\text {-Commanditaires } \\
\text {-Producteurs } \\
\text {-Chercheurs }\end{array}$ & $\begin{array}{l}\text {-Le chercheur élabore } \\
\text { l'ensemble de la dé- } \\
\text { marche et la maîtrise }\end{array}$ \\
\hline $\begin{array}{l}\text {-Selon les hypothèses } \\
\text { du chercheur, les be- } \\
\text { soins-problèmes qui se } \\
\text { posent dans une po- } \\
\text { pulation }\end{array}$ & $\begin{array}{l}\text {-Commanditaires } \\
\text {-Producteurs } \\
\text {-Chercheurs } \\
\text {-Quelques membres de } \\
\text { la population (notables } \\
\text { et décideurs institu- } \\
\text { tionnels) }\end{array}$ & $\begin{array}{l}\text {-Le chercheur élabore } \\
\text { l'ensemble de la dé- } \\
\text { marche et la maîtrise, } \\
\text { tout en opérant quel- } \\
\text { ques adaptations:comp- } \\
\text { te tenu! des apports de } \\
\text { certains éléments de } \\
\text { la population à laquelle } \\
\text { il a renvoyé des résul- } \\
\text { tats ou des informa- } \\
\text { tions partiels }\end{array}$ \\
\hline $\begin{array}{l}\text {-Les situations-problèmes } \\
\text { identifiées I dans ou } \\
\text { par un milieu donné } \\
\text {-Les besoins essentiels } \\
\text { ou fondamentaux }\end{array}$ & $\begin{array}{l}\text {-Chercheurs } \\
\text {-Commanditaires } \\
\text {-Animateurs } \\
\text {-Quelques collectifs de } \\
\text { la population (recon- } \\
\text { nus comme partenaires } \\
\text { valables) }\end{array}$ & $\begin{array}{l}\text {-Le collectif de cher- } \\
\text { cheurs élabore l'en- } \\
\text { semble de la démarche } \\
\text { (analyse et stratégie) } \\
\text { qu'il réajuste avec les } \\
\text { animateurs (après ré- } \\
\text { tro-alimentation) selon } \\
\text { les pressions, résis- } \\
\text { tances, obstacles ve- } \\
\text { nant de la population }\end{array}$ \\
\hline $\begin{array}{l}\text {-Les conflits dans la } \\
\text { réalité vécue par une } \\
\text { population à travers la } \\
\text { perception qu'elle en } \\
\text { a et son histoire col- } \\
\text { lective (conflits, reflets } \\
\text { des contradictions dans } \\
\text { la société) }\end{array}$ & $\begin{array}{l}\text {-Groupes militants en- } \\
\text { dogènes, dont au moins } \\
\text { un ayant un projet } \\
\text { socio-politique clair } \\
\text {-Population } \\
\text {-Animateurs militants }\end{array}$ & $\begin{array}{l}\text {-Les groupes militants } \\
\text { élaborent et maîtrisent } \\
\text { toutes les étapes du } \\
\text { processus } \\
\text {-Décodification par la } \\
\text { population des résul- } \\
\text { tats de l'enquête (qui } \\
\text { lui sont restitués sous } \\
\text { forme de code) et mo- } \\
\text { bilisation de cette po- } \\
\text { pulation sur une action } \\
\text { à mener }\end{array}$ \\
\hline $\begin{array}{l}\text {-Tensions et mythes } \\
\text { de mobilisation dans } \\
\text { la réalité quotidienne } \\
\text { et l'environnement du } \\
\text { groupe/communauté }\end{array}$ & $\begin{array}{l}\text {-Chercheurs militants } \\
\text {-Groupe moteur }\end{array}$ & $\begin{array}{l}\text {-Choix du groupe d'in- } \\
\text { tervention par le cher- } \\
\text { cheur qui élabore les } \\
\text { hypothèses, récolte les } \\
\text { données, les systéma- } \\
\text { tise et les restitue à la } \\
\text { communauté en terme } \\
\text { d'opposition aux mo- } \\
\text { dèles surtout culturels }\end{array}$ \\
\hline
\end{tabular}


ANNEXE 3 (suite)

\begin{tabular}{|c|c|}
\hline Type de projet & Objectifs prioritaires \\
\hline $\begin{array}{l}\text { Élaboration de projets modélisés ou } \\
\text { le plus souvent "marketing" }\end{array}$ & $\begin{array}{l}\text {-Recueil d'information } \\
\text {-Développement de la production } \\
\text { de documents ou d'ouvrages }\end{array}$ \\
\hline $\begin{array}{l}\text { Adaptation de la population con- } \\
\text { cernée à un projet prédéterminé }\end{array}$ & $\begin{array}{l}\text {-Accumulation de faits pour véri- } \\
\text { fier les hypothèses des chercheurs } \\
\text {-Production de documents ou d'ou- } \\
\text { vrages } \\
\text {-Élaboration de projets de déve- } \\
\text { loppement }\end{array}$ \\
\hline $\begin{array}{l}\text { Réforme par la population d'un } \\
\text { projet dont la politique générale } \\
\text { est prédéterminée }\end{array}$ & $\begin{array}{l}\text {-Articulation: Recherche/Action } \\
\text { Pratique/Théorie } \\
\text { Chercheurs/Praticiens } \\
\text {-Rapport et évaluation de l'expé- } \\
\text { rience }\end{array}$ \\
\hline $\begin{array}{l}\text { Projet de lutte autonome, élaboré, } \\
\text { mis en place par les groupes mili- } \\
\text { tants porteurs dont l'impact s'accroît } \\
\text { dans la population }\end{array}$ & $\begin{array}{l}\text {-Regard critique sur la réalité } \\
\text {-Formation politique à partir du } \\
\text { processus de conscientisation } \\
\text {-Élaboration d'un nouveau proces- } \\
\text { sus d'acquisition de connaissances } \\
\text {-Mobilisation populaire }\end{array}$ \\
\hline $\begin{array}{l}\text { Mise en route d'un processus per- } \\
\text { manent de dénonciation/action/ } \\
\text { réflexion }\end{array}$ & $\begin{array}{l}\text {-Saisir le potentiel de changement } \\
\text { à l'intérieur de chaque situation } \\
\text { et actualiser ce potentiel } \\
\text {-Actions possibles, réalistes, ca- } \\
\text { pables d'améliorer la vie quoti- } \\
\text { dienne }\end{array}$ \\
\hline
\end{tabular}


ANNEXE 3 (suite)

\begin{tabular}{|c|c|c|}
\hline Visées prioritaires & $\begin{array}{l}\text { Degré d'implication } \\
\text { et de solidarité des } \\
\text { chercheurs/animateurs } \\
\text { dans la situation } \\
\text { d'enquête }\end{array}$ & $\begin{array}{c}\text { Dangers } \\
\text { spécifiques }\end{array}$ \\
\hline $\begin{array}{l}\text {-Avancement et accu- } \\
\text { mulation des sciences } \\
\text { (sociales ou techniques) } \\
\text { et de leurs applications } \\
\text { opérationnelles }\end{array}$ & $\begin{array}{l}\text {-Distance préservée de } \\
\text { l'expert }\end{array}$ & $\begin{array}{l}\text {-Maintien des popula- } \\
\text { tions dans une situa- } \\
\text { tion de passivité et d'a- } \\
\text { liénation } \\
\text {-Pouvoir accru des ex- } \\
\text { perts et des comman- } \\
\text { ditaires }\end{array}$ \\
\hline $\begin{array}{l}\text {-Avancement qualita- } \\
\text { tif des sciences (sociales } \\
\text { ou techniques) et de } \\
\text { leurs applications opé- } \\
\text { rationnelles } \\
\text {-Développement du } \\
\text { consensus sur des axes } \\
\text { du changement social }\end{array}$ & $\begin{array}{l}\text {-Réduction de la dis- } \\
\text { tance chercheurs/ani- } \\
\text { mateurs/population }\end{array}$ & $\begin{array}{l}\text {-Renforcement du pou- } \\
\text { voir et de la crédibilité } \\
\text { des notables, ainsi que } \\
\text { du collectif de cher- } \\
\text { cheurs }\end{array}$ \\
\hline $\begin{array}{l}\text { - Lutte contre une trop } \\
\text { rigide vision du travail } \\
\text { (fonctions et tâches) } \\
\text {-Création d'une nou- } \\
\text { velle situation d'ap- } \\
\text { prentissage } \\
\text {-Appui au changement } \\
\text { social }\end{array}$ & $\begin{array}{l}\text {-Implication plus ou } \\
\text { moins grande des cher- } \\
\text { cheurs/animateurs dans } \\
\text { les problèmes de la } \\
\text { population, le proces- } \\
\text { sus éducatif engagé ou } \\
\text { dans l'action définie }\end{array}$ & $\begin{array}{l}\text {-Reproduction moder- } \\
\text { nisée des rapports so- } \\
\text { ciaux } \\
\text {-Réponse immédiate } \\
\text { aux situations-problèmes } \\
\text { et non action sur les } \\
\text { causes structurelles }\end{array}$ \\
\hline $\begin{array}{l}\text {-Analyse de la réalité } \\
\text { (locale, régionale, na- } \\
\text { tionale) } \\
\text {-Stratégies de luttes } \\
\text { (avec identification des } \\
\text { enjeux à court, moyen } \\
\text { et long terme) } \\
\text {-Libération des exploi- } \\
\text { tations économiques, } \\
\text { des dominations socio- } \\
\text { politiques des aliéna- } \\
\text { tions culturelles et idéo- } \\
\text { logiques }\end{array}$ & $\begin{array}{l}\text {-Solidarité concrète } \\
\text { des partenaires, non } \\
\text { seulement dans le pro- } \\
\text { cessus commun d'édu- } \\
\text { cation politique, mais } \\
\text { encore dans l'engage- } \\
\text { ment pour la transfor- } \\
\text { mation des structures } \\
\text { et des mentalités }\end{array}$ & $\begin{array}{l}\text {-Mauvaise évaluation } \\
\text { des capacités réelles } \\
\text { des groupes militants } \\
\text { demandeurs } \\
\text {-Manipulation de la } \\
\text { population par le guide } \\
\text { d'interview ou le ques- } \\
\text { tionnaire } \\
\text {-Découragement des } \\
\text { militants devant la len- } \\
\text { teur du processus }\end{array}$ \\
\hline $\begin{array}{l}\text {-Éducation idéologique } \\
\text { à partir du décalage } \\
\text { repéré entre la situa- } \\
\text { tion réelle du groupe } \\
\text { et la perception qu'il } \\
\text { en a } \\
\text {-Appropriation par les } \\
\text { groupes opprimés du } \\
\text { "savoir scientifique" } \\
\text { pour l'utiliser,comme } \\
\text { outil de libération }\end{array}$ & $\begin{array}{l}\text {-Reconnaissance réci- } \\
\text { proque de la solidari- } \\
\text { té entre chercheurs/ } \\
\text { avant-garde et com- } \\
\text { munauté }\end{array}$ & $\begin{array}{l}\text {-Leadership intellectuel } \\
\text { et idéologique } \\
\text {-Non mise en place } \\
\text { d'un projet collectif et } \\
\text { efficace à long terme }\end{array}$ \\
\hline
\end{tabular}

